\title{
Minimal Access Surgical Evacuation of Unilateral Chronic Subdural Hematoma
}

\author{
Hamdy Mohammed Behairy \\ Department of Neurosurgery, Al-Azhar University, Cairo, Egypt \\ Email: dr.hamdybehairy@hotmail.com
}

How to cite this paper: Behairy, H.M. (2018) Minimal Access Surgical Evacuation of Unilateral Chronic Subdural Hematoma. World Journal of Neuroscience, 8, 82-89. https://doi.org/10.4236/wins.2018.81007

Received: December 18, 2017

Accepted: January 29, 2018

Published: February 1, 2018

Copyright $\odot 2018$ by author and Scientific Research Publishing Inc. This work is licensed under the Creative Commons Attribution International License (CC BY 4.0).

http://creativecommons.org/licenses/by/4.0/

\begin{abstract}
Chronic subdural hematoma (CSDH) is one of the most common neurosurgical conditions occurring mostly in elderly and ideally treated with surgical drainage. Many surgical techniques of different degrees of invasiveness have been proposed for its treatment with controversy about the best surgical procedure. The study aims to evaluate the efficacy of the minimal access technique of single burr-hole craniostomy and massive irrigation for treatment of unilateral CSDH. The study is a prospective descriptive study involved all patients with symptomatic radiologically proven unilateral CSDH admitted and treated surgically in department of Neurosurgery, Al-Hussein University hospital, Al-Azhar University, Cairo, Egypt, over 3 years, from January 2013 to January 2016. In the results we found that total 64 patients of CSDH, treated surgically by this procedure. Men formed $72 \%$ and women $28 \%$. Age range was 51 to 82 years; the mean age was 65 years. Clinical presentation was impaired consciousness in $7 \%$, headache $(59 \%)$, right hemiparesis in $56 \%$ and left hemiparesis in 37\%. CT was done for $91 \%$ and MRI $27 \%$ patients. The hematoma was left in $61 \%$ and right in $39 \%$. Surgical evacuation utilizing this procedure was done in all patients. Complete recovery was obtained in $82 \%$ of patients and partial recovery in $11 \%$ of patients. Follow up for 4 months was done. Recurrence was detected in $6 \%$ of patients. We concluded that unilateral CSDH can be treated effectively by single nondependent burr-hole craniostomy and massive irrigation with excellent outcome.
\end{abstract}

\section{Keywords}

Chronic Subdural Hematoma, Burr-Hole Evacuation

\section{Introduction}

Chronic subdural hematoma (CSDH) is one of the most common types of intra- 
cranial hematomas especially in elderly people after minor head trauma [1]. CSDH is dark altered fluid blood, aged more than 3 weeks [2] and appears as crescentic subdural collection, iso, mixed or hypodense in CT scan and predominantly hyperintense in MRI [3] [4]. Risk factors include head trauma which is mostly minor, advanced age due to brain atrophy that leads to widening of subdural space and stretching the bridging fragile subdural veins, more tendency to fall and the usual antiplatelet administration [5]. Possible factors that enlarge $\mathrm{CSDH}$ over time are osmotic gradient of hemolytic products, low oncotic pressure inside the hematoma capsule, repeated micro-hemorrhaging, anticoagulant and profibrinolytic materials, inflammatory and growth factors role [6]. So, wash of subdural blood through irrigation is able to abolish all these factors that predispose to recurrence. Markwalder et al., (1981) presented clinical classification of CSDH as follows: Grade 0: asymptomatic, Grade 1: alert, oriented, mild symptoms (e.g. headache), Grade 2: drowsy or disoriented, variable neurological deficits (e.g. hemiparesis), Grade 3: stupor but responds to stimuli, severe focal signs (e.g. hemiplegia) and Grade 4: coma, flexes/extends to pain [7]. Computerized tomography (CT) scan is rapid and convenient for all cases. CT findings include brain atrophy, mid-line shift, crescentic shape subdural haematoma of different densities and location mostly in the fronto-parietal or fronto-parieto-occipital convexity. Most CSDHs are hypodense in CT but may be isodense or of mixed density due to layers of blood and membranes. Isodense CSDHs are better evaluated by MRI to get more detailed internal structure of the hematoma as size, septae and membranes with hyperintensity in both T1 and T2 sequences [4]. Surgical treatment has been widely accepted as the most effective management method for CSDH. The initial surgical technique is controversial and a standard one does not exist [8]. Several surgical techniques ranging from just twist drill tapping and aspiration to more invasive procedures including single burr-hole drainage, enlarged burr-hole (small craniectomy), multiple burr-holes drainage, and complex craniotomy (with or without membranectomy) (with or without subgaleal or subdural closed drainage system) were used. There is correlation between the rate of recurrence and the surgical technique employed [9]. An Indian study in 2005 reported success of treatment of CSDH with single burr hole craniostomy and irrigation [10]. Meta-analysis of 631 patients found no significant difference in recurrence rates related to the number of burr holes used and decided that single burr hole craniostomy appears as good as double burr hole craniostomy [11]. So, I have performed this study to evaluate the efficacy of utilizing less invasive minimal access surgical steps aiming to get highest benefits within least time and with least possible or without complications. The steps included single burr hole craniostomy and sustained irrigation till clear saline get back and no drains was inserted. An Indian study performed the same technique and reported excellent outcome [12]. Air water tightness was obtained by making the burr hole nondependent and the highest point during surgery and temporary plugging it with gel foam after 
completing the irrigation, tightening sutures that was made and kept stay before burr hole drilling and healing that occurs better with non-leaking wound. In this study we assumed postoperative lesion side up posture to maintain air water tightness till healing seals the wound.

\section{Material and Methods}

This is a descriptive prospective study included all patients with symptomatic radiologically proven (by CT scan and/or MRI) as CSDH treated surgically in department of neurosurgery, Al-Hussein university hospital, Al-Azhar university, Cairo, Egypt, over 3 years, from January 2013 to January 2016. Patients of any age and sex were included provided that the hematoma was unilateral and the patient was managed surgically through single burr hole evacuation. Bilaterally affected patients or patients not subjected to surgery whatever the cause and those with acute and subacute hematomas and cases complicating ventricular shunting were excluded. Also patients treated through two burr holes as in clearly polyseptate hematomas were also excluded from the study. Patients were evaluated at time of admission through full history, physical examination, laboratory investigation including coagulation parameters and imaging (CT and/or MRI). An informed written consent was assigned by the patient and patient's caring relative. The technique used for all cases under general anaesthesia was single nondependent burr hole craniostomy made over the maximum thickness of the hematoma in the posterior frontal/parietal region. Prolonged irrigation of the subdural space with warm normal saline using $50 \mathrm{ml}$ syringes gently till clear saline comes back. The flow and dilution effect of controlled gentle pressure and circumferential irrigation eliminate the altered thick fluid blood, profibrinolytic compounds and air from subdural space and help in breaching of septae and removal of far off clots along with some membrane shreds. It is necessary to minimize irrigation pressure and jet to avoid injury of cortical vessels and the membrane covering brain surface then complete filling the hematoma cavity with saline was done while the burr hole was looking up without drainage, with temporary sealing the burr hole with gel foam and water tight single layer skin closure through tightening early formed loose stay stitches.

Single burr hole craniostomy often takes shorter time and less tissue invasiveness. Choosing nondependent site of the burr hole was to help maximum drainage and maximum benefit from irrigation with avoiding premature closure of the burr hole by the re-expanding brain in dependent one. Also leakage of saline filling the hematoma cavity and consequently air entrance which may form pneumocephalus in dependent burr hole is avoided.

A necessary step is to perform early stay sutures of the skin before forming the burr hole with meticulous hemostasis of skin and galia where at the end of the operation, just tightening of the sutures is needed without puncturing the skin which is frequently precipitates acute recurrence from unnoticed bleeding scalp arteries. I preferred the lateral head position with surgery site up intraoperative 
as well as in the few hours postoperative to prevent saline extrusion through the burr hole and leakage through the wound which is still not sealed by early healing. Good hydration early postoperative helps brain re-expansion. After 24 hours, out of bed mobilization of the patient was allowed. Patients were discharged after 2 - 5 days according to the clinical condition.

For all patients, suture removal was done after 10 - 12 days in the minor surgery room inside the operative theatre section under complete aseptic conditions to avoid risk of dragging introduction of infection followed by sterile redressing for 3 days to be sure of sealing of suture puncture sites.

The last patient was operated upon 4 months before the end of the study which spent 3 years then assessment of the results of the study was done. All patients were followed-up in neurosurgical outpatient-clinic (clinically and by CT scan if needed) for at least 4 times over 4 months (after 1 week, 3 weeks, 1 month and 2 months) with urgent consultation at any time if symptoms suggestive recurrence were found where CT was done and if recurrence was detected, re-admission was done for frequent aspiration in the operative theatre under complete aseptic conditions without anaesthesia while the head was in lateral position and the wound site was the highest point. A medium gouge cannula was introduced about $1.5 \mathrm{~cm}$ away from wound line in valve like technique till just passing the inner bone edge of the burr hole where the trucker of the cannula was removed and the blood came out without suction or irrigation.

\section{Results}

This is a descriptive prospective study performed over 3 years, from January 2013 to January 2016 in department of neurosurgery, Al-Hussein university hospital, Al-Azhar university, Cairo, Egypt and included 64 patients with symptomatic radiologically proven (by CT scan and/or MRI) unilateral chronic subdural hematoma (CSDH) who were admitted and treated surgically. The 64 patients involved 46 men (72\%) and 18 women (28\%) with male predominance $(\mathrm{M}: \mathrm{F}=7: 3)$. Their ages ranged from 51 to 82 years (Table 1 ).

The mean age of them was 65 years. Sixty two patients were right handed (97\%) and 2 patients were left handed (3\%). Definite minor head trauma of was mentioned by 16 patients (25\%) 2 - 6 weeks before presentation while in 48 patients (75\%) no definite trauma was mentioned. The clinical presentation was deterioration of consciousness level in 4 patients (7\%), headache in 38 patients (59\%)

Table 1. Showing the demographic data of study population.

\begin{tabular}{ccc}
\hline Age range & \multicolumn{2}{c}{ Sex } \\
\cline { 2 - 3 } & Males & Females \\
\hline $51-61$ years & 9 & 4 \\
$62-72$ years & 32 & 11 \\
$73-82$ years & 6 & 3 \\
$51-82$ years & $\mathbf{4 6}$ & $\mathbf{1 8}$ \\
\hline
\end{tabular}


and right hemiparesis of varying degree and aphasia in 36 patients (56\%) and left hemiparesis in 24 patients (37\%). CT was done for 58 patients (91\%) including all patients presented with consciousness deterioration and all patients with severe symptoms. MRI was done as the only radiological investigation for 6 cases (9\%) with mild clinical deficits while in 12 patients (18\%), MRI was done following CT scan where mixed density was revealed to get more radiological details especially hematoma compartmentation by septa or membranes.

The haematoma was commoner on left than on right side. It was left in $39 \mathrm{pa}-$ tients (61\%) and right in 25 patients (39\%). The hematoma was fronto-parietal in 43 patients (67\%) and fronto-parieto-occipital in 21 patients (33\%) (Table 2).

Under general anaesthesia, single burr hole evacuation was performed in all patients $(100 \%)$ in the posterior frontal/parietal nondependent region which together with temporary closure of burr hole with gel foam and water tight closure of the wound without drain and postoperative lateral position of head with wound site up kept saline filling hematoma cavity in place in order not to leak and replaced by air with development of pneumocephalus. The last patient was operated upon 4 months before the end of the study. All patients were followed-up in neurosurgical outpatient-clinic (clinically and by CT scan if needed) for at least 4 times over 4 months (after 1 week, 3 weeks, 1 month and 2 months) with urgent consultation at any time if symptoms suggestive recurrence were found. Only 10 patients (15\%) followed the follow up schedule tightly while 54 patients $(85 \%)$ presented for follow up irregularly. The clinical outcome was excellent. Complete recovery of symptoms and signs was obtained within 2 days in 42 patients $(65 \%)$ and within 5 days in 11 patients (17\%) while partial recovery was obtained within 5 days in 7 patients (11\%). The primarily unconscious 4 patients regained full consciousness in $2-4$ days followed by $2-5$ days care then discharge with residual weakness and incomplete brain re-expansion (Table 3 ).

No pre- or post-operative seizures occurred in all patients and no anticonvalscents were given. Radiological follow up (CT scan) was done in the 11 patients $(17 \%)$ with partial recovery $(7+4)$ and 4 patients $(6 \%)$ came back within 2 weeks postoperative with suspected recurrence where subdural mixed density was found indicating recurrence and needed readmission and frequent aspiration with final complete cure within 2 weeks. No cases of mortality. Complications including intra cerebral hematoma, formation of contralateral subdural hematoma or subdural empyema were not reported in this study. Hospital stay

Table 2. Shows incidence of situation of chronic subdural hematoma when unilateral.

\begin{tabular}{ccc}
\hline Site of the hematoma (CT/MRI) & No. of cases & $\%$ \\
\hline Left fronto-parietal & 22 & $34 \%$ \\
Right fronto-parietal & 21 & $33 \%$ \\
Left fronto-parieto-occipital & 17 & $27 \%$ \\
Right fronto-parieto-occipital & 4 & $6 \%$ \\
Total & 64 & $100 \%$ \\
\hline
\end{tabular}


Table 3. Showing examples of pre- and post-operative images for study cases.

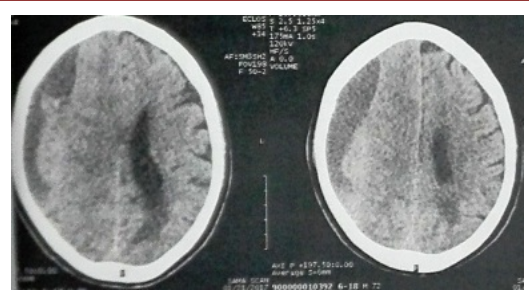

1A: preoperative CT, Rt. Fronto-parietal Hypodense CSDH

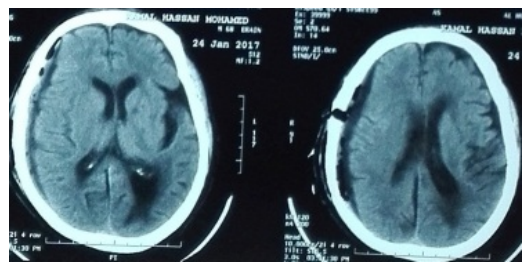

1B: postoperative CT Re-expanding brain

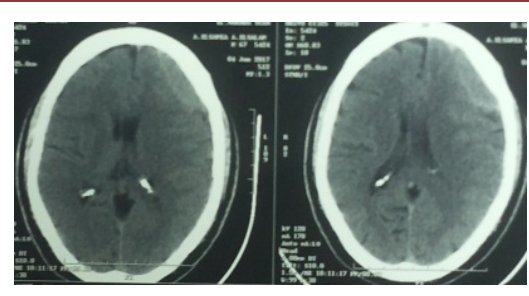

2A: preoperative CT, Lt. Fronto-parietal isodense $\mathrm{CSDH}$

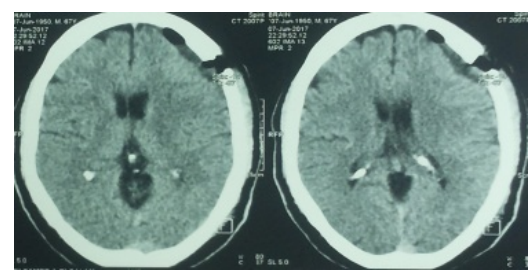

2B: postoperative CT Re-expanded brain

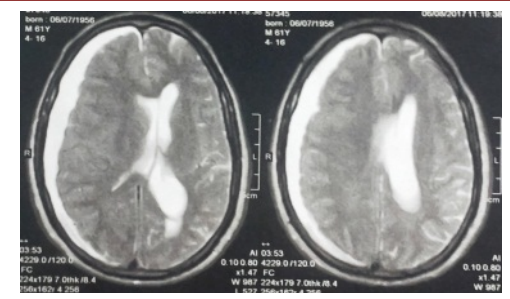

3A: preoperative MRI, Rt. Fronto-parieto-occipital Hyperintense CSDH

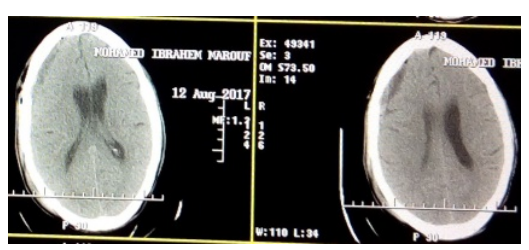

3B: postoperative CT Re-expanded brain

was 2 to 5 days in primarily fully conscious patients and after regaining full consciousness in primarily unconscious patients. Patients with recurrence readmitted and stayed 2 weeks before discharge.

\section{Discussion}

In this study, men formed $72 \%$ while women formed $28 \%$ of patients indicating male predominance in affection with $\mathrm{CSDH}$ because out-door activities is much more and extend to old ages in males than females in Egyptian society. It was reported in a similar study that males represented $85 \%$ [13]. The ages ranged from 51 to 82 years indicating that the 5 th decade onwards is a risk factor because of wide subdural space due to brain atrophy, fragile stretched subdural veins and frequent minor trauma. Tripathy et al., (2013) mentioned that 52.8\% of the patients in his study were between 55 - 74 years age range [12]. It was reported that the mean age of cases affected with CSDH was 64.9 years [14].

Clinically, only $25 \%$ of patients mentioned definite minor head trauma 2 - 6 weeks before onset of symptoms. It was reported that the aetiology in their study was identifiable in $56 \%$ patients who had a history of head trauma and the interval between the trauma and onset of symptoms varied from 14 to 72 days [4].

This study was mainly concerned with results of performing simplest minimal access surgical steps in management of unilateral CSDH through single burr hole craniostomy. A similar study reported that Single burr-hole with saline lavage technique is an adequate and safe treatment for $\mathrm{CSDH}$ and putting two holes in this pathology mayn't always be necessary [12]. Also, many studies that compared single and double-burr-holes groups reported that the difference in outcome and recurrence rates was not statistically significant [11] [15] [16]. The rate of recurrence in this study was $6 \%$ which is an acceptable one. A similar study reported recurrence rate of $8 \%-26 \%$ [17]. In this study, clinical outcome 
was excellent with complete recovery within 2 days in $65 \%$ and within 5 days in $17 \%$ while partial recovery was obtained within 5 days in $11 \%$. No cases of mortality. Complications including post operatively intra cerebral hematoma, formation of contralateral subdural hematoma or subdural empyema were not reported in this study may be due to highly aseptic meticulous practice. Performing larger studied with larger number of patients is recommended for support of the results.

\section{Conclusion}

Unilateral chronic subdural hematoma can be dealt with adequately and effectively utilizing minimal access single nondependent burr hole craniostomy, gentle massive irrigation and refilling the hematoma cavity with saline without subdural drainage with good outcome with advantages of short operating time, easy and simple procedure with very low recurrence and mortality rate.

\section{Acknowledgements}

For all my colleagues of department of neurosurgery, Al-Azhar University.

\section{Conflicts of Interest}

There are no conflicts of interest.

\section{Financial Support and Sponsorship}

Nil.

\section{References}

[1] Matsumoto, K., Akagi, K., Abekura, M., Ryujin, H., Ohkawa, M. and Iwasa, N. (1999) Recurrence Factor for Chronic Subdural Haematomas after Burr-Hole Craniostomy and Closed System Drainage. Neurological Research, 21, 277-280. https://doi.org/10.1080/01616412.1999.11740931

[2] Macfarlane, M., Weerakkody, Y. and Kathiravel, Y. (2009) Chronic Subdural Haematomas Are Common on Left Than on the Right. Journal of Clinical Neuroscience, 16, 642-644. https://doi.org/10.1016/j.jocn.2008.07.074

[3] Osborn, A. (1994) Diagnostic Neuroradiology. Mosby, St. Louis, 205-211.

[4] Senturk, S., Guzel, A., Bilici, A., Takmaz, I., Guzel, E. and Aluclu, U. (2010) CT and MR Imaging of Chronic Subdural Haematomas: A Comparative Study. Swiss Medical Weekly, 140, 335-340.

[5] Chen, J. and Levy, M. (2000) Causes, Epidemiology, and Risk Factors of Chronic Subdural Hematoma. Neurosurgery Clinics of North America, 11, 399-406.

[6] Baechli, H., Nordmann, A., Bucher, H. and Gratzl, O. (2004) Demographics and Prevalent Risk Factors of Chronic Subdural Haematoma: Results of a Large Single-Center Cohort Study. Neurosurgical Review, 27, 263-266. https://doi.org/10.1007/s10143-004-0337-6

[7] Markwalder, T., Steinsiepe, K., Rohner, M., Reichenbach, W. and Markwalder, H. (1981) The Course of Chronic Subdural Hematomas after Burr-Hole Craniostomy and Closed-System Drainage. Journal of Neurosurgery, 55, 390-396. 
https://doi.org/10.3171/jns.1981.55.3.0390

[8] Lee, J., Ebel, H., Ernestus, R. and Klug, N. (2004) Various Surgical Treatments of Chronic Subdural Hematoma and Outcome in 172 Patients: Is Membranectomy Necessary? Surgical Neurology, 61, 523-528.

https://doi.org/10.1016/j.surneu.2003.10.026

[9] Papacocea, T., Adam, D., Croitoru, R., Rusu, I. and Papacocea, A. (2016) Factors Influencing the Recurrence Rate of Operated Chronic Subdural Hematomas. Romanian Neurosurgery, 2, 162-167.

[10] Gurunathan, J. (2005) Treatment of Chronic Subdural Hematoma with Burr Hole Craniostomy and Irrigation. Indian Journal of Neurotrauma (IJNT), 2, 127-130.

[11] Belkhair, S. and Pickett, G. (2013) One versus Double Burr Holes for Treating Chronic Subdural Hematoma Meta-Analysis. Canadian Journal of Neurological Sciences, 40, 56-60. https://doi.org/10.1017/S0317167100012956

[12] Tripathy, S., Mishra, S., Mahapatra, A., Panda, R., Majhi, H. and Mishra, J. (2013) Role of "Single Burr-Hole and Saline Lavage" in Chronic Subdural Hematoma (csdh): The Need of Another Clinical Prospective Epidemiological Study. Journal of Neurology and Neuroscience, 6, 51.

[13] Sanjay, K. and Atul, J. (2014) Comparative Study of Evacuation of Chronic Subdural Haematoma by Two Burr Holes Technique versus Single Burr Hole with Partial Excision of Membrane Technique. International Journal of Medical Science and Education, 1, 107-112.

[14] Jeong, S., Kim, S. and Won, Y. (2014) Clinical Analysis of Risk Factors for Recurrence in Patients with Chronic Subdural Hematoma Undergoing Burr Hole Trephination. Korean Journal of Neurotrauma, 10, 15-21. https://doi.org/10.13004/kjnt.2014.10.1.15

[15] Asaduzzaman, S., Islam, K. and Hussein, M. (2014) Comparative Study between Single versus Double Burr-Hole Drainage of Unilateral Chronic Subdural Haematoma. Bangladesh Medical Journal, 43, 13-16.

[16] Nayil, K., Altaf, R., Shoaib, Y., Wani, A., Laharwal, M. and Zahoor, A. (2014) Chronic Subdural Hematomas: Single or Double Burr Hole-Results of a Randomized Study. Turkish Neurosurgery, 24, 246-248.

[17] Kansal, R., Nadkarni, T. and Goel, A. (2010) Single versus Double Burr Hole Drainage of Chronic Subdural Hematomas. A Study of 267 Cases. Journal of Clinical Neuroscience, 17, 428-429. https://doi.org/10.1016/j.jocn.2009.07.109 